\title{
Short-term effects of a combined nutraceutical of insulin-sensitivity, lipid level and indexes of liver steatosis: a double-blind, randomized, cross-over clinical trial
}

\author{
Arrigo FG Cicero ${ }^{1,2^{*}}$, Martina Rosticci ${ }^{1}$, Angelo Parini ${ }^{1}$, Martino Morbini ${ }^{1}$, Riccardo Urso ${ }^{1}$, Elisa Grandi \\ and Claudio Borghi ${ }^{1}$
}

\begin{abstract}
Background: Overweight subjects easily develop alterations of the glucose and lipid metabolism and are exposed to an increased cardiometabolic risk. This condition is potentially reversible through the improvement of dietary and behavioural habits. However, a well-assembled nutraceutical would be a useful tool to better improve the metabolic parameters associated to overweight and insulin resistance.

Methods: To evaluate the effect of a combined nutraceutical containing berberine, chlorogenic acid and tocotrienols, we performed a double blind, cross-over designed trial versus placebo, in 40 overweight subjects with mixed hyperlipidaemia. After the first 8 weeks of treatment (or placebo), patients were asked to observe a 2-week washout period, and they were then assigned to the alternative treatment for a further period of 8 weeks. Clinical and laboratory data associated to hyperlipidaemia and insulin resistance have been obtained at the baseline, at the end of the first treatment period, after the washout, and again after the second treatment period.

Results: Both groups experienced a significant improvement of anthropometric and biochemical parameters versus baseline. However, total cholesterol, LDL cholesterol, triglycerides, non-HDL cholesterol, fasting insulin, HOMA-IR, GOT and Lipid Accumulation Product decreased more significantly in the nutraceutical group versus placebo.

Conclusions: This combination seems to improve a large number of metabolic and liver parameters on the short-term in overweight subjects. Further studies are needed to confirm these observations on the middle- and long-term.
\end{abstract}

Keywords: Berberine, Chlorogenic acid, Nutraceutical, Insulin-sensitivity, Lipoproteins, Liver steatosis

\section{Background}

Overweight and obesity are a worldwide leading health problem [1], especially when complicated by other diseases [2]. In particular, overweight is usually associated to a large number of metabolic changes, such as reduction of insulin-sensitivity, dyslipidaemia, accumulation of lipids in the liver, respectively predisposing to the

\footnotetext{
* Correspondence: afgcicero@cardionet.it

'Department of Medicine and Surgery Sciences, University of Bologna, Bologna, Italy

${ }^{2}$ Hypertension Research Center, Poliambulatorio Pad. 2, Via Albertoni, 15, 40138 Bologna, Italy
}

\section{Ciomed Central}

development of type 2 diabetes, atherosclerosis and nonalcoholic steatohepatitis, all conditions increasing the risk to develop cardiovascular disease [3,4]. All these changes are potentially reversible with intensification of physical activity and improvement of dietary habits [5], while the efficacy/safety ratio of the available pharmaceutical drugs is not yet adequately defined [6].

Recent literature shows that a number of natural compounds seem to have positive effects on the metabolic changes associated to overweight $[7,8]$. Of particular interest seems to be soluble fibres, cinnamon, chromium 
picolinate, polyunsaturated fatty acids, berberine, chlorogenic acid, green tea, bitter gourd and banaba.

The main limitation in the clinical use of these products are the need to assume large quantities (for instance, cinnamon and bitter gourd), the scarce availability of clinical data (for instance, banaba), the relatively small metabolic effect (for instance, fibres and chromium), the need to use low doses in order to avoid side effects (for instance, berberine), and the cost (for instance, high quality green tea) [9]. On the other side, scarce data are available as it regards the clinical efficacy of combination of these substances.

In this context, the aim of our study was to evaluate the short-term metabolic effects of a combined nutraceutical containing berberine and chlorogenic acid on overweight subjects with mixed hyperlipidemia.

\section{Methods and materials}

\section{Study design}

This double blind, placebo controlled, randomized clinical trial was carried out in 40 moderately hypercholesterolemic subjects with metabolic syndrome, in primary prevention for cardiovascular diseases, consecutively enrolled in the ambulatory service of cardiovascular disease prevention in the Medical and Surgical Sciences Department of the University of Bologna.

The study consisted in a cross-over designed trial of 18 weeks, preceded by 4 weeks of diet stabilization in which patients received standard behavioural and qualitative (not quantitative) dietary suggestions to correct unhealthy habits. In particular, subjects were instructed to follow a general indication of a Mediterranean diet, avoiding excessive intake of dairy products and red meat derived products during the study, and maintaining overall constant dietary habits. Individuals were also generically encouraged to increase their physical activity by walking briskly for 20 to 30 minutes, 3 to 5 times per week, or by cycling.

Inclusion criteria were:

- age between 25 and 75

- LDL-Cholesterol (LDL-C) level between 130 and $190 \mathrm{mg} / \mathrm{dl}$

- Body Mass Index (BMI) value between 25 and $30 \mathrm{~kg} / \mathrm{m}^{2}$

- Triglycerides (TG) level between 150 and $400 \mathrm{mg} / \mathrm{dl}$

- Fasting Plasma Glucose (FPG) level under 125 mg/dl.

- When present, assumption of hypolipidaemic or antihypertensive drugs stabilized since at least 6 months.

\section{Exclusion criteria were:}

- secondary prevention for cardiovascular diseases

- assumption of antidiabetic drugs
- inconstant or not-stabilized assumption of drugs which may interfere with lipid or glucose metabolism

- chronic gastrointestinal diseases and assumption of drugs for their treatment

- known thyroid, liver, renal or muscle diseases

- known allergy or intolerance to a component of the tested product

- any medical or surgical condition which could lead to an inconstant adhesion to the protocol.

The study was fully conducted in accordance with the Declaration of Helsinki, its protocol was approved by the Ethical Committee of the University of Bologna, and informed consent was obtained from all patients before the inclusion in the study.

\section{Treatments}

After 4 weeks of diet and physical activity stabilization, patients were allocated to treatment with an indistinguishable pill of placebo or with an active product $\left(\right.$ Trixy $\left.^{\circ}\right)$ containing Berberine from Berberis aristata, Tocotrienols form Elaeis guineensis and Chlorogenic acid form decaffeinated Green coffee (Coffea canephora), kindly offered by Nathura s.r.l. (Montecchio Emilia, RE).

After 8 weeks of treatment (or placebo), patients were asked to observe a 2-week wash-out period, and they were then assigned to the alternative treatment for a further period of 8 weeks. Clinical and laboratory data have been obtained at the baseline, at the end of the first treatment period, after the wash-out, and again after the second treatment period.

Randomization was done assigning an alphabetical code to each lot code (corresponding to treatment or placebo) impressed on the pillbox. Codes were then kept in a sealed envelope which was not opened until the end of the trial. Pillboxes were then mixed, and the investigators assigned a blinded pillbox to each enrolled patient.

Throughout the study, we instructed patients to take the product first dose on the day after they were given the study product. At the same time, all unused products were retrieved to the investigator.

\section{Assessments}

Personal data, CVD history and pharmaceutical anamnesis of each patient were inquired at the beginning of the trial. Anthropometrical parameters, such as body weight, waist circumference and BMI were collected both at the beginning and during the trial, together with hemodynamic and biochemical parameters. BMI was calculated as weight in kilograms divided by the square of height in meters. Hemodynamic parameters were collected measuring orthostatic and clinostatic systolic and diastolic blood pressure (mean value of 3 interval measures). Also orthostatic and 
clinostatic wrist blood pressure (mean value of 3 interval measures) and cardiac frequency were collected.

All biochemical parameters were obtained after a 12hour overnight fast. The following parameters were obtained or calculated through appropriate formulas: total cholesterol (TC) [10], high-density lipoprotein-cholesterol (HDL-C) [11], TG [12], LDL-C [13], non-HDL cholesterol (nonHDL-C), lipoprotein(a) (Lpa), FPG, fasting insulin, homeostatic model assessment of insulin resistance (HOMA-IR) [14], creatinine, Serum Uric Acid (SUA), liver transaminases (glutamic oxaloacetic transaminase, GOT; glutamate-pyruvate transaminase, GPT), Gamma Glutamyl-Transferase ( $\gamma \mathrm{GT}$ ), Lipid Accumulation Product (LAP) and Hepatic Steatosis Index (HSI) [15]. All measurements were centrally performed in the laboratory of our department.

HOMA-IR is the simplest and most commonly used method to estimate insulin resistance. It was calculated as the product of basal glucose $(\mathrm{mmol} / \mathrm{l})$ and fasting insulin $(\mu \mathrm{U} / \mathrm{ml})$ divided by 22.5 [14].

LAP is calculated through the following formulas: (waist circumference $(\mathrm{cm})-65) \times(\mathrm{TG}(\mathrm{mmol} / \mathrm{l}))$ for men and: (waist circumference $(\mathrm{cm})-58) \times(\mathrm{TG}(\mathrm{mmol} / \mathrm{l}))$ for women, and is a useful marker of the individual cardiometabolic risk [16]. HSI, instead, is a simple index used to evaluate the presence or the severity of Non-alcoholic Fatty Liver Disease (NAFLD) through some biochemical parameters (TG, BMI, yGT, waist circumference) [16]. Adherence to behavioural counselling and to treatment/placebo, tolerability, acceptability and compliance were also assessed.

\section{Statistical analyses}

The main outcome of the study was to evaluate the different effect of the active treatment and placebo on
HOMA-index. Thus, we calculated the sample size of the study on the assumption that the HOMA-index standard deviation of the difference in the response variables is 0.7 , so that with 40 patients enrolled, the probability was 80 percent that the study would have detected a treatment difference at a two-sided 0.05 significance level, if the true difference between treatments is 0.318 units.

Data have been analysed using intention to treat by mean of the Statistical Package for Social Science (SPSS) 21.0, version for Windows. Normally distributed baseline characteristics of the population have been compared using Student's $t$ test, non-normally distributed parameters using Mann-Whitney- $U$ test. Two-way analyses of variance for crossover design were used to assess the effect of treatment during the assumption of placebo or treatment. All data are expressed as means and Standard Deviation (SD). To verify the basic assumptions of crossover design, besides the evaluation of period effect, the presence of a carryover effect was excluded. Level of statistical significance was set on $P=0.05$.

\section{Results}

The main characteristics of the enrolled patients have been resumed in Table 1. At the baseline the two treatment sequence group were balanced for all the investigated parameters. All the patients completed the study and no one experienced adverse events during the trial.

Compared to baseline values, no change has been observed after both treatments as it regards Diastolic Blood Pressure (DBP), Pulse Pressure (PP), Heart Rate (HR), GOT, HSI, creatinine and SUA level.

Both groups experienced a significant decrease in body weight, BMI and waist circumference $(\mathrm{p}<0.001)$, without

Table 1 Baseline characteristics of the subjects enrolled in the trial

\begin{tabular}{|c|c|c|c|c|c|}
\hline N. 40 & Mean & SD & & Mean & SD \\
\hline Age (years) & 53.45 & 8.00 & Total Cholesterol (mg/dL) & 215.70 & 15.20 \\
\hline Weight (kg) & 82.00 & 11.45 & LDL-C (mg/dL) & 132.15 & 17.50 \\
\hline Body Mass Index (kg/m2) & 28.75 & 2.65 & Triglycerides (mg/dL) & 222.05 & 38.70 \\
\hline Waist Circumference (cm) & 106.30 & 10.85 & $\mathrm{HDL}-\mathrm{C}(\mathrm{mg} / \mathrm{dL})$ & 39.10 & 3.40 \\
\hline $\mathrm{SBP}(\mathrm{mmHg})$ & 135.50 & 14.90 & Non HDL-C (mg/dL) & 176.60 & 15.40 \\
\hline $\mathrm{DBP}(\mathrm{mmHg})$ & 80.60 & 7.30 & GOT (U/L) & 24.90 & 10.10 \\
\hline Pulse Pressure (mmHg) & 54.90 & 12.10 & GPT (U/L) & 28.10 & 12.95 \\
\hline MAP $(\mathrm{mmHg})$ & 98.95 & 8.75 & Serum Uric Acid (mg/dL) & 4.50 & 1.30 \\
\hline Heart Rate (bpm) & 73.80 & 5.60 & Creatinine (mg/dL) & 8.95 & 1.25 \\
\hline $\mathrm{FPG}(\mathrm{mg} / \mathrm{dL})$ & 106.90 & 6.70 & LAP & 111.40 & 34.35 \\
\hline Insulin $(\mu \mathrm{U} / \mathrm{ml})$ & 14.50 & 4.30 & $\mathrm{HSI}$ & 37.05 & 3.60 \\
\hline HOMA-IR & 4.05 & 1.20 & & & \\
\hline
\end{tabular}

Acronyms: SBP: systolic blood pressure, DBP: diastolic blood pressure, MAP: mean arterial pressure, LDL-C: LDL cholesterol, HDL-C: HDL cholesterol, Non HDL-C: non HDL cholesterol, FPG: fasting plasma glucose, HOMA-IR: homeostatic model assessment of insulin resistance, GOT: glutamic oxaloacetic transaminase, GPT: glutamate-pyruvate transaminase, LAP: lipid accumulation product, HSI: hepatic steatosis index. 
significant difference between the treatments. Systolic and mean arterial pressure mildly, but significantly improved after diet-nutraceutical treatment. Lipid pattern improved after both treatments, however after diet-nutraceutical treatment TC, LDL-C, TG, and non-HDL-C decreased more significantly than after diet alone $(\mathrm{p}<0.001)$. After diet-nutraceutical treatment HDL-C also mildly, but significantly, increased versus baseline. FPG similarly decreased after both treatments, but fasting insulin and HOMA significantly decreased versus baseline values after diet-nutraceutical treatment only: the reduction was also significant versus diet-placebo ( $\mathrm{p}<0.001$ for both HOMAIR and insulin). Then, GOT improved versus baseline and versus diet-placebo $(\mathrm{p}<0.001)$ after diet- nutraceutical treatment. Finally, LAP improved after both treatments, but the reduction after diet-nutraceutical treatment was significantly higher than the one obtained with dietplacebo $(\mathrm{p}=0.021)($ Table 2$)$.

The wash-out did not determine a significant carryover effect in both group, probably because its relatively short duration and because the improvement in dietary habits persisted during this phase. However, the group who began with diet-nutraceutical treatment, at the end of the study experience a regression of a large number of metabolic parameters (HOMA-index, TC, LDL-C, non HDL-C) to values no more significantly different than the baseline ones ( $p>0.05$ for all).

\section{Discussion}

The insulin-sensitizing property of coffee [15] has been partly attributed to its content in chlorogenic acid, an ester formed from cinnamic acids and quinic acid. In particular, chlorogenic acid seems to act as insulin sensitizer that potentiates insulin action similar to the therapeutic action of metformin [17]. However, recent data suggest that it could also exert its antidiabetic action by inhibiting $\alpha$-amylase and $\alpha$-glucosidase activities [18].

Moreover, the effect of chlorogenic acid on expression of hepatic peroxisome proliferator-activated receptoralpha [19] and on activation of $5^{\prime}$ AMP-activated protein kinase [20] partly explain the presumed liver protective effect of coffee from non-alcoholic fatty liver disease [21].

Table 2 Mean difference in changes of the studied parameters after placebo or nutraceutical treatment

\begin{tabular}{|c|c|c|c|c|}
\hline \multirow[t]{2}{*}{ N.40 } & \multirow{2}{*}{$\begin{array}{l}\text { Mean difference } \\
\text { in changes }\end{array}$} & \multicolumn{2}{|l|}{$95 \% \mathrm{Cl}$} & \multirow[t]{2}{*}{$P$} \\
\hline & & Lower & Upper & \\
\hline$\Delta$ Weight $(\mathrm{kg})$ & $-0,2$ & -0.9 & 1.1 & 0.534 \\
\hline$\Delta$ Body Mass Index (kg/m2) & $-0,1$ & -0.8 & 1.0 & 0.687 \\
\hline$\Delta$ Waist Circumference $(\mathrm{cm})$ & $-0,6$ & -1.1 & 1.2 & 0.432 \\
\hline$\triangle$ Systolic Blood Pressure (mmHg) & 0,2 & -0.6 & 0.8 & 0.326 \\
\hline$\Delta$ Diastolic Blood Pressure $(\mathrm{mmHg})$ & 0,8 & -0.1 & 1.3 & 0.091 \\
\hline$\triangle$ Pulse Pressure $(\mathrm{mmHg})$ & $-0,5$ & -0.9 & 0.2 & 0.084 \\
\hline$\Delta$ Mean Arterial Pressure $(\mathrm{mmHg})$ & 0,5 & -0.2 & 0.9 & 0.102 \\
\hline$\Delta$ Heart Rate (bpm) & $-0,1$ & -1.3 & 1.3 & 0.518 \\
\hline$\Delta$ Total Cholesterol (mg/dL) & $-19,5$ & -61.4 & -9.3 & $<0.001$ \\
\hline$\Delta \mathrm{LDL}-\mathrm{C}(\mathrm{mg} / \mathrm{dL})$ & $-15,4$ & -45.3 & -2.7 & $<0.001$ \\
\hline$\Delta$ Triglycerides (mg/dL) & $-21,4$ & -64.3 & -1.1 & $<0.001$ \\
\hline$\triangle \mathrm{HDL}-\mathrm{C}(\mathrm{mg} / \mathrm{dL})$ & 0,3 & -0.1 & 2.1 & 0.054 \\
\hline$\triangle$ Non HDL-C (mg/dL) & $-19,7$ & -62.2 & -3.0 & $<0.001$ \\
\hline$\Delta$ Fasting Plasma Glucose (mg/dL) & $-0,1$ & -9.4 & 7.6 & 0.433 \\
\hline$\Delta$ Insulin $(\mu \mathrm{U} / \mathrm{ml})$ & -1 & -1.1 & -0.1 & 0.018 \\
\hline$\triangle \mathrm{HOMA}-\mathrm{IR}$ & $-0,3$ & -0.5 & -0.1 & $<0.001$ \\
\hline$\triangle \mathrm{GOT}(\mathrm{U} / \mathrm{L})$ & $-0,3$ & -0.7 & 0.9 & 0.681 \\
\hline$\triangle \mathrm{GPT}(\mathrm{U} / \mathrm{L})$ & $-7,8$ & -11.4 & -0.9 & 0.027 \\
\hline$\Delta$ Serum Uric Acid (mg/dL) & $-2,4$ & -3.7 & 1.4 & 0.349 \\
\hline$\Delta$ Creatinine (mg/dL) & $-0,5$ & -0.9 & 0.8 & 0.441 \\
\hline$\Delta$ Lipid Accumulation Product & $-10,4$ & -44.5 & -0.3 & 0.002 \\
\hline$\Delta$ Hepatic Steatosis Index & 1,3 & -0.1 & 2.9 & 0.068 \\
\hline
\end{tabular}

Acronyms: LDL-C: LDL cholesterol, HDL-C: HDL cholesterol, Non HDL-C: non HDL cholesterol, HOMA-IR: homeostatic model assessment of insulin resistance, GOT: glutamic oxaloacetic transaminase, GPT: glutamate-pyruvate transaminase. 
Berberine has a well-known antihyperlipidaemic effect, both inhibiting the liver LDL-receptor inactivation by inhibiting PCSK9 and activating the $5^{\prime}$ AMP-activated protein kinase [22]. Moreover, berberine exerts insulinsensitizing effects with a metformin-like mechanism of action [23]. Recent data also suggest that berberine could also induce improvement in liver steatosis, probably as consequence of the relevant changes induced in triglycerides level and insulin-sensitivity [24].

So, as expected from the available literature data (1624), after a combined treatment with berberine and chlorogenic acid we observed a significant improvement in LDL-C $(-24 \%)$, TG $(-19 \%)$, non HDL-C $(-22 \%)$, fasting insulin $(-5 \%)$ and HOMA index $(-10 \%)$. Moreover, we also observed a significant improvement in LAP $(-25 \%)$, a validated index of liver steatosis [16].

All these effects could be particularly relevant in the management of overweight subjects complicated with metabolic syndrome and/or non-alcoholic fatty liver disease, both condition associated to high cardiometabolic disease risk [25,26] and whose management is actually mainly based on diet and life-style improvement [27,28].

This study has some limitation. The first one is the relatively small sample size, partially balanced by the cross-over design of the trial. The second one is the relatively short duration of the trial, however comparable with the most part of explorative trials carried out with metabolically active nutraceuticals. Then, we have not prescribed a specific diet based on bromatological data, but we gave only general dietary suggestion finalized at avoiding dietary excess in order to simulate more strictly a condition of general practice. Finally, we have not directly evaluated the degree of liver steatosis. On the other side, validated index of liver steatosis have been used, supported by recent literature. Finally, we did not compare the effects of berberine and chlorogenic acid alone and with their association, but the effect of the two components alone on the parameters investigated was already known in literature.

\section{Conclusions}

On the basis of our preliminary data, a nutraceutical containing berberine and chlorogenic acid seems to be well-tolerated and able to improve a large number of metabolic and liver parameters on the short-term in overweight patients with mixed hyperlipidaemia. Further studies are needed to confirm these observations on the middle- and long-term.

\section{Abbreviations}

BMI: Body mass index; DBP: Diastolic blood pressure; FPG: Fasting plasma glucose; GT: Gamma glutamyl-transferase; GOT: Glutamic oxaloacetic transaminase; GPT: Glutamate-pyruvate transaminase; HDL-C: High-density lipoprotein-cholesterol; HOMA-IR: HOmeostatic model assessment of insulin resistance; HR: Heart rate; HSI: Hepatic steatosis index; LAP: Lipid accumulation product; LDL-C: Low-density lipoprotein-cholesterol;
Lpa: Lipoprotein(a); MAP: Mean arterial pressure; NAFLD: Non-alcoholic fatty liver disease; nonHDL-C: nonhigh-density lipoprotein-cholesterol; PP: Pulse pressure; SBP: Systolic blood pressure; SD: Standard deviation; SUA: Serum uric acid; TC: Total cholesterol; TG: Triglycerides; WC: Waist circumference.

\section{Competing interests}

The authors declare that they have no competing interests. The founding sponsors had no role in the design of the study; in the collection, analyses, or interpretation of data; in the writing of the manuscript, and in the decision to publish the results.

\section{Authors' contributions}

AFGCicero designed the study protocol and carried out the statistical analysis. Then he wrote the paper with CB who also supervised the staff. MR, $A P$ and $R U$ screened and enrolled the patients, following them during the study. EG managed the blood samples and carried out the lab analyses. All the Authors read and approved the text of the paper.

\section{Acknowledgments}

We are sincerely grateful to Annalisa Culzoni for the attentive assistance and support in external independent data recheck and paper revision.

Received: 21 October 2014 Accepted: 17 March 2015

Published online: 28 March 2015

\section{References}

1. Ng M, Fleming T, Robinson M, Thomson B, Graetz N, Margono C, et al. Global, regional, and national prevalence of overweight and obesity in children and adults during 1980-2013: a systematic analysis for the Global Burden of Disease Study 2013. Lancet. 2014;384(9945):766-81.

2. Yang Z, Zhang N. The burden of overweight and obesity on long-term care and Medicaid financing. Med Care. 2014;52(7):658-63.

3. Bauer UE, Briss PA, Goodman RA, Bowman BA. Prevention of chronic disease in the 21st century: elimination of the leading preventable causes of premature death and disability in the USA. Lancet. 2014;384(9937):45-52.

4. Kones R, Rumana U. Prevention of cardiovascular disease: updating the immensity of the challenge and the role of risk factors. Hosp Pract. 2014;42(1):92-100.

5. Ebbert JO, Elrashidi MY, Jensen MD. Managing overweight and obesity in adults to reduce cardiovascular disease risk. Curr Atheroscler Rep. 2014;16(10):445.

6. Rueda-Clausen CF, Padwal RS. Pharmacotherapy for weight loss. BMJ. 2014;348:g3526.

7. Magrone T, Perez de Heredia F, Jirillo E, Morabito G, Marcos A, Serafini M. Functional foods and nutraceuticals as therapeutic tools for the treatment of diet-related diseases. Can J Physiol Pharmacol. 2013;91(6):387-96.

8. Conroy KP, Davidson IM, Warnock M. Pathogenic obesity and nutraceuticals. Proc Nutr Soc. 2011;70(4):426-38.

9. Cicero AF, Tartagni E, Ertek S. Nutraceuticals for metabolic syndrome management: from laboratory to benchside. Curr Vasc Pharmacol. 2014;12(4):565-71.

10. Klose $\mathrm{S}$, Borner K. Enzymatic determination of total cholesterol with the Greiner Selective Analyzer (GSA II). J Clin Chem Clin Biochem. 1977;15:121-30.

11. Havel RJ, Eder HA, Bragdon JH. The distribution and chemical composition of ultracentrifugally separated lipoproteins in human serum. J Clin Invest. 1955:34:1345-53

12. Wahlefeld AW. Triglycerides determination after enzymatic hydrolysis. In: Bermeyer H, editor. Methods of Enzymatic Analysis. 2nd English ed. New York: Academic Press; 1974. p. 18-31.

13. Friedewald WT, Levy RI, Fredrickson DS. Estimation of the concentration of low density lipoprotein cholesterol in plasma, without use of the preparative ultracentrifuge. Clin Chem. 1972;18:499-502.

14. Matthews DR, Hosker JP, Rudenski AS, Naylor BA, Treacher DF, Turner RC. Homeostasis model assessment: insulin resistance and beta-cell function from fasting plasma glucose and insulin concentrations in man. Diabetologia. 1985:28:412-9.

15. van Dam RM, Feskens EJM. Coffee consumption and risk of type 2 diabetes mellitus. Lancet. 2002;360(9344):1477-8.

16. Cicero AFG, D'Addato S, Reggi A, Marchesini Reggiani G, Borghi C, on behalf of the Brisighella Heart Study. Hepatic Steatosis Index and Lipid Accumulation Product as middle-term predictors of incident metabolic 
syndrome in a large population sample: data from the Brisighella Heart Study. Intern Emerg Med. 2013;8(3):265-7.

17. McCarty MF. A chlorogenic acid-induced increase in GLP-1 production may mediate the impact of heavy coffee consumption on diabetes risk. Med Hypotheses. 2005;64(4):848-53.

18. Oboh G, Agunloye OM, Adefegha SA, Akinyemi AJ, Ademiluyi AO. Caffeic and chlorogenic acids inhibit key enzymes linked to type 2 diabetes (in vitro): a comparative study. J Basic Clin Physiol Pharmacol. 2015;26(2):165-70.

19. Li SY, Chang CQ, Ma FY, Yu CL. Modulating effects of chlorogenic acid on lipids and glucose metabolism and expression of hepatic peroxisome proliferator-activated receptor-alpha in golden hamsters fed on high fat diet. Biomed Environ Sci. 2009;22(2):122-9.

20. Ong KW, Hsu A, Tan BK. Anti-diabetic and anti-lipidemic effects of chlorogenic acid are mediated by ampk activation. Biochem Pharmacol. 2013;85(9):1341-51.

21. Chen S, Teoh NC, Chitturi S, Farrell GC. Coffee and non-alcoholic fatty liver disease: brewing evidence for hepatoprotection? I Gastroenterol Hepatol. 2014;29(3):435-41.

22. Cicero AFG, Ertek S. Metabolic and cardiovascular effects of berberine: from preclinical evidences to clinical trial results. Clin Lipidol. 2009;4(5):553-63.

23. Derosa G, Maffioli P, Cicero AFG. Berberine on metabolic and cardiovascular risk factors: an analysis from preclinical evidences to clinical trials. Expert Opin Biol Ther. 2012;12(8):1113-24.

24. Cicero AFG, Derosa G, Maffioli P, Reggi A, Parini A, Rosticci M, et al. Berberine induced improvement in Hepatic Steatosis Index in overweight dyslipidaemic patients treated with lipid-lowering nutraceuticals. Curr Topics Nutr Res. 2013;11(1-2):41-6.

25. Kaur J. A comprehensive review on metabolic syndrome. Cardiol Res Pract. 2014;2014:943162.

26. Gruben N, Shiri-Sverdlov R, Koonen DP, Hofker MH. Nonalcoholic fatty liver disease: A main driver of insulin resistance or a dangerous liaison? Biochim Biophys Acta. 2014;1842(11):2329-43.

27. Cicero AF, Dormi A, D'Addato S, Gaddi AV, Borghi C. Long-term effect of a dietary education program on postmenopausal cardiovascular risk and metabolic syndrome: the Brisighella Heart Study. J Womens Health. 2010;19(1):133-7.

28. Nseir W, Hellou E, Assy N. Role of diet and lifestyle changes in nonalcoholic fatty liver disease. World J Gastroenterol. 2014;20(28):9338-44.

\section{Submit your next manuscript to BioMed Central and take full advantage of:}

- Convenient online submission

- Thorough peer review

- No space constraints or color figure charges

- Immediate publication on acceptance

- Inclusion in PubMed, CAS, Scopus and Google Scholar

- Research which is freely available for redistribution 